\title{
Abbe sine condition and related imaging conditions in geometrical optics
}

Joseph Braat

Joseph J. M. Braat, "Abbe sine condition and related imaging conditions in geometrical optics," Proc. SPIE 3190, Fifth International Topical Meeting on Education and Training in Optics, (8 December 1997); doi:

$10.1117 / 12.294417$

Event: Fifth International Topical Meeting on Education and Training in Optics, 1997, Delft, Netherlands 
The Abbe sine condition and related imaging conditions in geometrical optics

\author{
Joseph Braat \\ Philips Research Laboratories \\ Professor Holstlaan 4, 5656 AA Eindhoven, The Netherlands \\ Optics Research Group \\ Department of Applied Physics, Delft University of Technology \\ Lorentzweg 1, 2628 CJ Delft, The Netherlands
}

\begin{abstract}
Abbe's sine condition or other imaging conditions for the aperture rays of an axial pencil determine how an object volume is imaged by the optical system in image space. We analytically derive the imaging condition which should lead to the largest possible volume in image space where the aberration (spherical aberration and coma) stays below a certain prescribed level.
\end{abstract}

Keywords: geometrical optics, aberration, imaging

\title{
1. INTRODUCTION
}

The aberration theory of images generated by an optical system has been gradually developed from the seventeenth century on. Early analyses by e.g. Descartes, Roberval and Huygens concern the spherical aberration which arises when imaging an object point through a single refracting or reflecting surface. From this work we know the perfect or stigmatic imaging properties of conic sections (quadratic surfaces) and the so-called oval surfaces of Descartes (quartic surfaces). As a special case, the stigmatic points generated by a spherical surface are obtained (Huygens' aplanatic points). Further research was oriented towards the spherical aberration generated by a single lens and, later in the eighteenth century, the comatic aberration of off-axis pencils of rays. As a result, at the beginning of the nineteenth century, the as-designed quality of telescope objectives by Fraunhofer was very good (well within the diffraction limit) and the actual optical quality of the instruments was mainly limited by manufacturing imperfections and material inhomogeneities. However, the numerical aperture and the field angle of these objectives were rather small.

The need for an aberration theory valid for more complicated optical systems with larger values of numerical aperture and field angle was felt when photography emerged. The so-called third order aberration theory which, in principle, covers both larger aperture and field angles was probably first developed by Petzval but his results remained unpublished. A comprehensive third order theory for optical systems with circular symmetry consisting of an arbitrary sequence of optical elements was published by $L$. von Seidel in 1856. As a rule of thumb, this approximate theory yields reliable results for numerical apertures up to 0.10 or even 0.15 while the field angle may amount to some ten degrees. The Seidel third order theory was of great help for the design of photographic objectives with the specifications of the second half of the past century. For the design of e.g. microscope objectives with a high numerical aperture $(\geq 0.50)$ and a small field angle, the Seidel theory is not sufficient although it can produce a good design starting point.

In 1863, R. Clausius ${ }^{1}$ published a paper in which he shows under what condition an optical system (e.g. a mirror system) is able to concentrate radiant power in an optimum way even when the aperture of the imaging pencils is large. In 1874, H. Helmholtz ${ }^{2}$ showed that for correct imaging of a finite size object the so-called sine condition should be respected because otherwise the optical throughput or etendue of the optical system is not preserved from object to image space. But it is the name of $\mathrm{E}$. Abbe ${ }^{3}$ which is generally associated with this sine condition because he was the first to establish the link between this condition and the freedom of comatic aberration of the imaging system even at large aperture. Apart from the sine condition which is of importance for the sharp imaging of a flat object, there is the so-called Herschel condition ${ }^{4}$ which guarantees sharp imaging when the magnification is varied,

Telephone: ++31 40 2743112; Fax: ++31 40 2744335; e-mail: braat@natlab.research.philips.com 
i.e. when the object point is shifted along the optical axis.

In this paper we first give, in the second section, a short derivation of the sine condition and the Herschel condition. In the third section we address the question how to create the largest possible volume in image space where the aberration does not exceed a certain value.

\section{DERIVATION OF THE VARIOUS IMAGING CONDITIONS}

\subsection{The Abbe and the Herschel condition}

In Figure 1 we schematically show an optical system with its entrance and exit pupil located at $E$ and $E^{\prime}$. From a pencil of rays leaving the object point $P$ only a certain ray $P D D^{\prime} P^{\prime}$ is shown with direction cosines $(0, M, N)$ and $\left(0, M^{\prime}, N^{\prime}\right)$ in respectively the object and image space ( $D$ and $D^{\prime}$ are located on the pupil reference spheres). The plane of the drawing is the $y z$-plane with $x=0$. We suppose that the pencil of rays is exactly focused at $P^{\prime}$ (stigmatic imaging). We now want to know how a pencil of rays leaving a neighbouring object point $Q$ is focused in image space. According to paraxial optics, the position of an image point $Q^{\prime}$ derived from the object point $Q$, which has been subjected to infinitesimal shifts $\delta y$ and $\delta z$ with respect to $P$, is found by applying the correct (paraxial) magnification factors. The lateral magnification factor is denoted by $\beta^{\prime}=\delta y^{\prime} / \delta y$ and the axial magnification factor

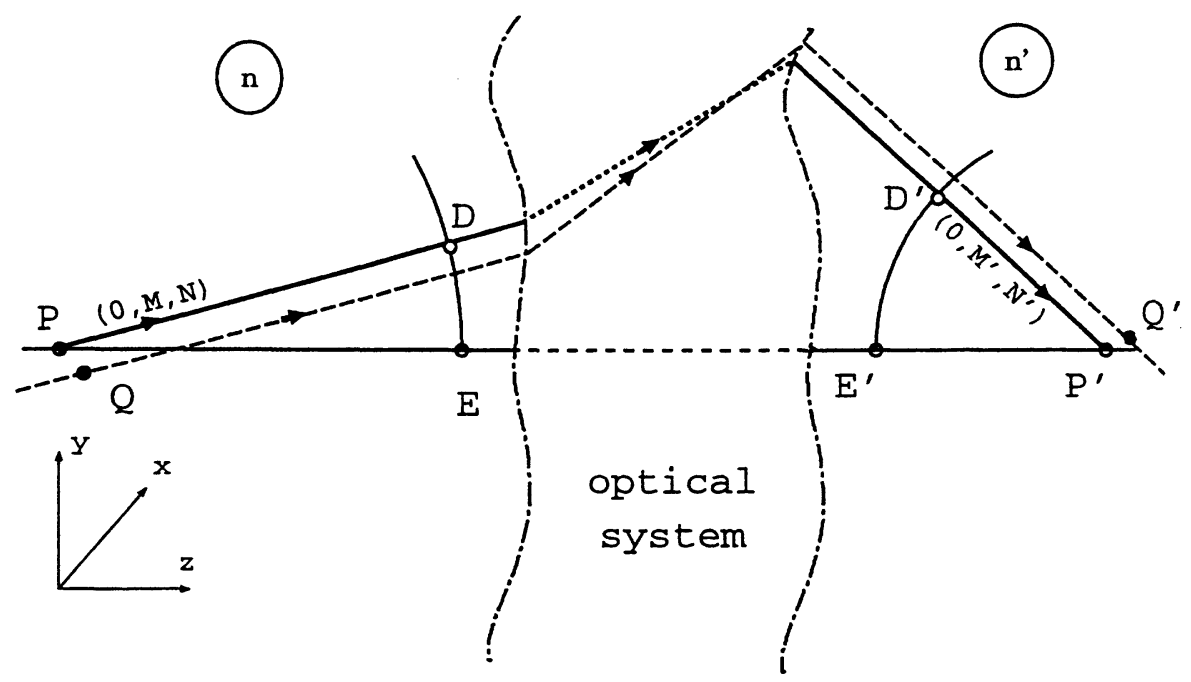

Figure 1. Schematic drawing illustrating the directions of two related rays in object and image space (the unknown trajectory through the optical system has been indicated by the dotted line). The image $P^{\prime}$ of $P$ is perfect. A second object point $Q$ has been shifted over infenitesimal distances $\delta z$ and $\delta y$ with respect to $P$. Its paraxial image is denoted by $Q^{\prime}$. The refractive indices of object and image space are denoted by $n$ and $n^{\prime}$.

is $\delta^{\prime}=\delta z^{\prime} / \delta z$. It can be shown that the relationship between the lateral and axial magnification is given by ${ }^{5}$

$$
\delta^{\prime}=\frac{n^{\prime}}{n} \beta^{\prime 2},
$$

where $n$ and $n^{\prime}$ are the refractive indices in object and image space.

It now has to be seen under what condition the image quality of $Q^{\prime}$ can be equal to the (perfect) quality of $P^{\prime}$. To this goal, we take, from the pencil of rays through $Q$, a particular ray (dashed in the figure) which is parallel to the ray through $P$ shown in the drawing. Physically spoken, we consider this ray to represent a small part of a plane wave with the direction cosines $(0, M, N)$. If the reference point for measuring pathlength is shifted form $P$ towards $Q$, the change $\Delta W$ in path of the propagating wave disturbance along the ray from $P$ to $P^{\prime}$ is given by the scalar product of the ray vector $\vec{s}=(0, M, N)$ and the displacement vector $\overrightarrow{\delta r}=(0, \delta y, \delta z)$ multiplied with the refractive index of the object space:

$$
\Delta W=-n \overrightarrow{\delta r} \cdot \vec{s} .
$$


The minus sign is needed here because a shift of $Q$ in the positive $z$-direction leads to shorter optical path for the ray along the optical axis.

In the image space, given the image displacement vector $\delta \vec{r}^{\prime}$ and the ray vector $\overrightarrow{s^{\prime}}$, we observe a path difference $\Delta W^{\prime}$ according to

$$
\Delta W^{\prime}=n^{\prime} \delta \vec{r}^{\prime} \cdot \vec{s}^{\prime} \text {. }
$$

Equal imaging quality in $Q$ and $P$ (isoplanatism) is obtained when the residual $\delta W$ of the path differences in object and image space is zero for arbitrary values of the ray vectors of all rays belonging to the object and image space pencils $\left(\delta W=\Delta W+\Delta W^{\prime}=0\right)$.

We discern two particular cases:

- $\delta \vec{r}=(0, \delta y, 0)$

The isoplanatic condition now becomes:

$$
\delta W_{C}=n^{\prime} \delta y^{\prime} M^{\prime}-n \delta y M=0 .
$$

Using the paraxial magnification which exists between $\delta y^{\prime}$ and $\delta y$ we obtain for the residual aberration

$$
\delta W_{C}=\left[M^{\prime}-\frac{n M}{n^{\prime} \beta^{\prime}}\right]\left(n^{\prime} \delta y^{\prime}\right) .
$$

This condition, which guarantees the absence of aberration if an infinitesimal lateral excursion off-axis is applied, is generally known as Abbe's sine condition. We have used the index $C$ for the aberration because this aberration is called coma.

- $\delta \vec{r}=(0,0, \delta z)$

The corresponding isoplanatic condition becomes:

$$
\delta W_{S}=n^{\prime} \delta z^{\prime} N^{\prime}-n \delta z N=0 .
$$

Using the paraxial axial magnification $\delta^{\prime}$ existing between $\delta z$ and $\delta z^{\prime}$ we obtain for the residual aberration:

$$
\delta W_{S}=\left\{N^{\prime}-\frac{n^{2} N}{n^{\prime 2} \beta^{\prime 2}}\right\}\left(n^{\prime} \delta z^{\prime}\right) .
$$

This condition, which guarantees an extended axial range over which the object point can be shifted, is known as Herschel's condition. The subscript $S$ has been used because the aberration which could appear is circularly symmetric spherical aberration.

In general, the constant pathlength difference $\left(n^{\prime} \delta z^{\prime}-n \delta z\right)$ encountered for the axial ray $\left(N=N^{\prime}=1\right)$ is subtracted from the expression above and we obtain:

$$
\delta W_{S}=\left\{\left(N^{\prime}-1\right)-\frac{n^{2}(N-1)}{n^{\prime 2} \beta^{\prime 2}}\right\}\left(n^{\prime} \delta z^{\prime}\right)=0 .
$$

Abbe's sine condition is a prerequisite for an optical system which needs to image an extended flat object, e.g. for a photographic camera objective, a reduction objective but also for a microscope objective or an astronomical telescope. Herschel's condition is required when a system needs to operate at different magnifications, e.g. a narrow field telescope for both remote and close observation. Unfortunately, both conditions seriously conflict when the numerical aperture becomes high. A particular case arises when $\beta^{\prime}= \pm n / n^{\prime}$, a case which reduces to unit magnification when $n=n^{\prime}$; here, both conditions can be satisfied simultaneously. In most applications, it is the sine condition which will prevail because otherwise the useful lateral or angular image field would become unacceptably small.

\subsection{Residual aberration}

In this subsection we calculate the residual aberration when either the Abbe or the Herschel condition is satisfied by the optical system. Either in the lateral (Abbe) or in the axial (Herschel) direction, the first derivative of the aberration with respect to the shift of the image point is zero but in the orthogonal direction, the residual aberration will generally increase rapidly. 


\subsubsection{Residual spherical aberration when the Abbe condition is satisfied}

When the Abbe sine condition is complied with, the relationship between the $M$-direction cosines is

$$
\frac{n M}{n^{\prime} \beta^{\prime}}=M^{\prime}
$$

Using the relation

$$
N=\sqrt{1-M^{2}}=\sqrt{1-\left(\frac{n^{\prime}}{n} \beta^{\prime} M^{\prime}\right)^{2}}
$$

and expanding the square root up to the fourth order in $M^{\prime}$ one obtains for the spherical aberration (Eq. 7 ) up to the fourth order:

$$
\delta W_{S}=\frac{1}{8}\left(\frac{n^{\prime 2} \beta^{\prime 2}}{n^{2}}-1\right) M^{\prime 4}\left(n^{\prime} \delta z^{\prime}\right)
$$

Applied to a microscope objective with a numerical aperture of 0.65 (NA $=M_{m}^{\prime}=$ maximum value of $M^{\prime}$ ) and a magnification $\beta^{\prime}=0$, we obtain a permitted excursion $\delta z^{\prime}$ equal to $24.6 \mu \mathrm{m}\left(n=n^{\prime}=1\right.$ and $\left.\lambda=0.55 \mu \mathrm{m}\right)$. For the permitted spherical aberration $W_{S}^{m}$ we take the value of $1 \lambda$ peak-to-valley, the tolerance limit for an optical system to be just diffraction-limited. ${ }^{6}$ When the numerical aperture is increased to 0.90 , the $z^{\prime}$-excursion reduces to $6.7 \mu \mathrm{m}$.

\subsubsection{Residual coma when the Herschel condition is satisfied}

If the Herschel condition is complied with, Eq. 6 holds and, after the elimination of $M, N$ and $N^{\prime}$, the expression for the comatic aberration (Eq. 5) yields up to the fifth order

$$
\delta W_{C}=-\frac{1}{8}\left(1-\frac{n^{\prime 2} \beta^{\prime 2}}{n^{2}}\right)\left\{M^{\prime 3}+\frac{M^{\prime 5}}{2}\right\}\left(n^{\prime} \delta y^{\prime}\right) .
$$

Using the same numerical values as above for a microscope objective and applying the just diffraction-limited aberration tolerance $W_{C}^{m}$ for coma of the third order $(0.63 \lambda)$, we obtain a maximum lateral excursion $\delta y_{m}^{\prime}$ of $10.0 \mu \mathrm{m}$ at an aperture of 0.65 . When the aperture is increased to 0.90 , the half field size $\delta y_{m}^{\prime}$ reduces to $3.8 \mu \mathrm{m}$.

A comparison of both imaging conditions shows that, especially at high aperture, the sine condition should be preferably imposed as the design criterion of the optical system. If the opposite choice (Herschel condition) is made, the lateral extent of the image field is unacceptably small. Note that the value of $\beta=0$ is the worst regarding the incompatibility of the Abbe and Herschel condition (we suppose $\left|\beta^{\prime}\right| \leq n / n^{\prime}$ ). The residual aberration gradually diminishes to zero when $\beta^{\prime}$ moves towards the optimum value $\pm n / n^{\prime}$.

\section{IMAGING CONDITION FOR MAXIMUM IMAGE VOLUME}

In this section we calculate the imaging condition which guarantees the largest possible volume in image space. The possible aberrations (spherical aberration and coma) should remain below their just diffraction limited values indicated above. For this maximum image volume to be obtained, we suppose that neither the Herschel nor the Abbe condition is fully satisfied.

\subsection{The axial and lateral extent of the imaging volume}

As a starting point, we tolerate an offence against the sine condition of the lowest (third) order according to:

$$
\delta W_{C}=\left(M^{\prime}-\frac{n M}{n^{\prime} \beta^{\prime}}\right)\left(n^{\prime} \delta y^{\prime}\right)=-\epsilon M^{\prime 3}\left(n^{\prime} \delta y^{\prime}\right) \leq W_{C}^{m},
$$

where $\epsilon$ is a small factor to be optimized further on.

With this comatic aberration, the maximum image field radius becomes

$$
\delta y_{m}^{\prime}=\frac{W_{C}^{m}}{|\epsilon| n^{\prime} M_{m}^{\prime 3}}
$$


with $M_{m}^{\prime}$ the maximum aperture of the optical system.

The corresponding value of the spherical aberration is given by Eq. (7); the elimination of $M, N$ and $N^{\prime}$ is done with the aid of the expressions

$$
\begin{array}{r}
M^{\prime}-\frac{n M}{n^{\prime} \beta^{\prime}}=-\epsilon M^{\prime 3} \\
N \approx \sqrt{1-M^{2}}=1-\frac{M^{2}}{2}-\frac{M^{4}}{8} \\
N^{\prime} \approx \sqrt{1-M^{\prime 2}}=1-\frac{M^{\prime 2}}{2}-\frac{M^{\prime 4}}{8} .
\end{array}
$$

After some algebra, the resulting spherical aberration turns out to be given by:

$$
\delta W_{S}=\left[\epsilon+\frac{1}{8}\left(\frac{n^{\prime 2} \beta^{\prime 2}}{n^{2}}-1\right)\right] M^{\prime 4}\left(n^{\prime} \delta z^{\prime}\right) .
$$

The maximum axial excursion $\delta z_{m}^{\prime}$ now becomes

$$
\delta z_{m}^{\prime}=\frac{W_{S}^{m}}{n^{\prime} M_{m}^{\prime 4}\left[\epsilon+\frac{1}{8}\left(\frac{n^{\prime 2}{\beta^{\prime}}^{2}}{n^{2}}-1\right)\right]} .
$$

For the still diffraction-limited image volume with the shape of an ellipsoid we find the expression

$$
\begin{aligned}
\delta V & =\left|\frac{4 \pi}{3}\left(\delta y_{m}^{\prime}\right)^{2} \delta z_{m}^{\prime}\right| \\
& =\frac{4 \pi}{3}\left(\frac{W_{C}^{m}}{\epsilon n^{\prime} M_{m}^{\prime 3}}\right)^{2}\left|\frac{W_{S}^{m}}{n^{\prime} M_{m}^{\prime 4}\left[\epsilon+\frac{1}{8}\left(\frac{n^{\prime 2}{\beta^{\prime 2}}^{2}}{n^{2}}-1\right)\right]}\right|
\end{aligned}
$$

The substitution of the values for $W_{C}^{m}(0.63 \lambda)$ and $W_{S}^{m}(1 \lambda)$ yields the final expression

$$
\delta V=1.67\left\{\frac{\lambda^{3}}{n^{\prime 3} M_{m}^{\prime 10}}\right\} \cdot\left\{\frac{1}{\epsilon^{2}\left[\epsilon+\frac{1}{8}\left(\frac{n^{\prime 2} \beta^{\prime 2}}{n^{2}}-1\right)\right]}\right\} .
$$

\subsection{The maximum possible image volume}

To maximize the expression for the just diffraction-limited image volume $\delta V$, the denominator of the second term in Eq. 19 should be minimized. In Figure 2 we have depicted the $\epsilon$-dependent function

$$
f(\epsilon)=\left\{\epsilon^{2}\left[\epsilon+\frac{1}{8}\left(\beta^{\prime 2}-1\right)\right]\right\}
$$

in the denominator of Eq. 19 for several values of the lateral magnification $\beta^{\prime}\left(n=n^{\prime}=1\right)$.

The values $\epsilon=0$ and $\epsilon=\left(1-\beta^{\prime 2}\right) / 8$ correspond to respectively the Abbe and Herschel condition. However, these conditions do not necessarily guarantee a maximum image volume for a real optical system when the linear approximation for the aberration change is not sufficient.

An interesting point certainly is the extremum found at the value

$$
\epsilon_{e x t r}=\frac{1}{12}\left(1-\frac{n^{\prime 2} \beta^{2}}{n^{2}}\right) .
$$

This extremum is gradually shifting to the origin when the magnification approaches the value $\left|n / n^{\prime}\right|$, corresponding to the specific case where both the Abbe and Herschel condition can be satisfied simultaneously.

The numerical values associated with the extremum considered above are: 


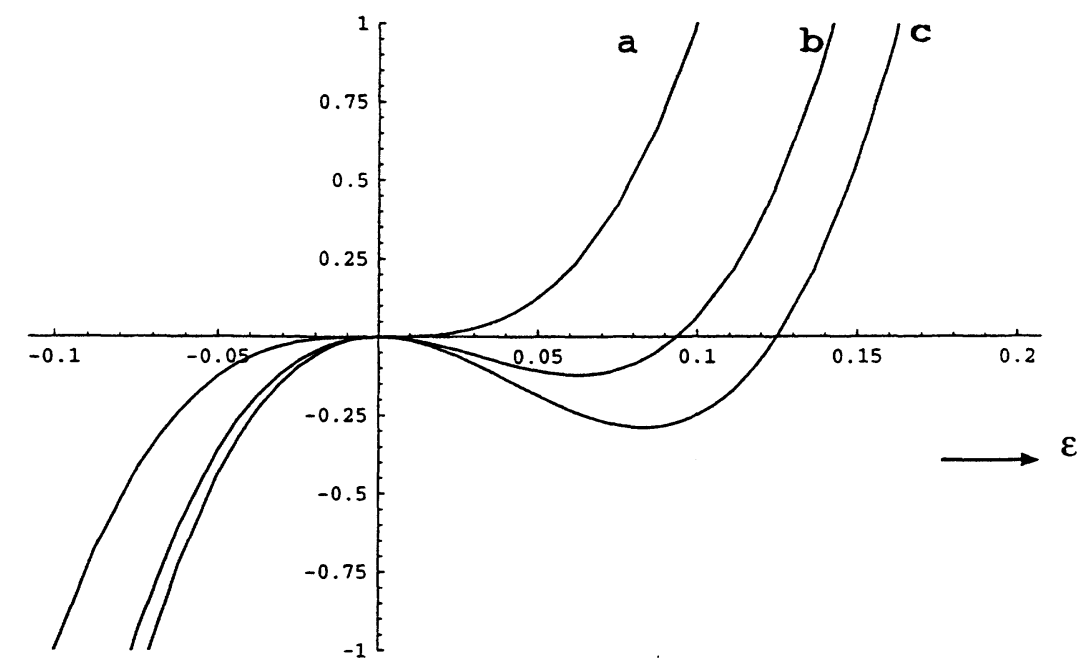

Figure 2. The behaviour of the function $10^{3} \cdot f(\epsilon)$ to be minimized for maximum image volume. The lateral magnification factor $\beta^{\prime}$ is the parameter and equals 1 (curve a), 0.5 (curve b) and 0 (curve c).

- $\beta^{\prime}=0, \lambda=0.55 \mu \mathrm{m}, n=n^{\prime}=1, \mathrm{NA}=M_{m}^{\prime}=0.65$ :

$\delta y^{\prime}=15.1 \mu \mathrm{m} \quad \delta z^{\prime}=73.9 \mu \mathrm{m}$

- idem, $\mathrm{NA}=0.90$

$\delta y^{\prime}=5.7 \mu \mathrm{m} \quad \delta z^{\prime}=20.1 \mu \mathrm{m}$

For the actual volume available at the value $\epsilon_{\text {extr }}$ we obtain $\left(n=n^{\prime}=1\right)$

$$
\delta V_{e x t r}=\frac{5745 \lambda^{3}}{\left(1-\beta^{\prime 2}\right)^{3} M_{m}^{\prime 10}}=\frac{4 \pi}{3}\left\{\frac{11.1 \lambda}{\left(1-\beta^{\prime 2}\right)}\right\}^{3} \frac{1}{M_{m}^{\prime 10}}
$$

\section{CONCLUSION}

We have studied the different possibilities to design an optical system regarding the directional properties of the rays of the axial pencil. Apart from the specific cases, known for a long time from the literature, we have analyzed the imaging condition which should lead to, e.g., a maximum diffraction-limited image volume. The obtained results should be checked by real-world designs in order to verify the validity of the first order analysis.

The design of an optical system towards maximum imaging volume is of practical importance in, among others, 3D scanning confocal microscopy.

\section{REFERENCES}

1. R. Clausius, "Ueber die Concentration von Wärme- und Lichtstrahlen und die Grenzen ihrer Wirkung," Annalen der Physik und Chemie 121, No. 1, pp. 1-44, 1864.

2. H. Helmholtz, "Die theoretische Grenze für die Leistungsfähigkeit der Mikroskope," Annalen der Physik und Chemie, pp. 557-584, 1874.

3. E. Abbe, "Beiträge zur Theorie des Mikroskops und der mikroskopischen Wahrnehmung," Archiv fuer mikroskopische Anatomie 9, pp. 413-468, 1873.

4. J. Herschel Phil. Trans. Roy. Soc. 111, pp. 222-266, 1821.

5. W. Welford, Aberrations of optical systems, Adam Hilger, Bristol, 1986.

6. M. Born and E. Wolf, Principles of Optics, Pergamon Press, New York, 1980. 